\title{
Qualidade de Sono, desejos alimentares e marcadores bioquímicos de inflamação em profissionais de Saúde
}

\author{
Sleep Quality, Food Cravings and Biochemical Markers of Inflammation in Health Professionals \\ Calidad del sueño, antojos de alimentos y marcadores bioquímicos de inflamación en profesionales \\ de la salud
}

Recebido: 28/10/2021 | Revisado: 30/10/2021 |Aceito: 30/10/2021 | Publicado: 31/10/2021

Marta Maria da Silva Lira Batista

ORCID: https://orcid.org/0000-0001-5995-0535 Universidade Federal do Piauí, Brasil Hospital Universitário do Piauí, Brasil

E-mail: fgamarthalira@gmail.com

Raimunda Sheyla Carneiro Dias

ORCID: https://orcid.org/0000-0003-1851-9495

Hospital Universitário do Piauí, Brasil

E-mail: sheylak75@yahoo.com.br

Gabrielly Martins de Barros

ORCID: https://orcid.org/0000-0001-9696-424X

Hospital Universitário do Piaú, Brasil

E-mail: gabriellymb95@gmail.com

Gleydyson Wesley Freire Lima

ORCID: https://orcid.org/0000-0003-3204-4473

Universidade Federal do Piauí, Brasil

E-mail: gwfreire@hotmail.com

Jaynara Keylla Moreira da Silva

ORCID: https://orcid.org/0000-0002-8043-3716

Nutricionista Clínica, Brasil

E-mail: jaynarakeylla@gmail.com

Maria Rita Carvalho Silva

ORCID: https://orcid.org/0000-0003-2270-6736

Faculdade de Ensino Superior do Piauí, Brasil

E-mail: mariaritacarvalho_@outlook.com

Mycaele Aline Santana Sousa

ORCID: https://orcid.org/0000-0001-5654-5905

Hospital Universitário do Piauí, Brasil

E-mail: micele29@gmail.com

Paulo César dos Santos

ORCID: https://orcid.org/0000-0001-7391-0337

Universidade Federal do Rio Grande do Norte, Brasil

E-mail: cesar.santos@ebserh.gov.br

Raianny Katiucia da Silva

ORCID: https://orcid.org/0000-0003-0439-0811

Hospital de Urgências de Teresina, Brasil

E-mail: raiannykatiucia28@gmail.com

Maria do Carmo de Carvalho e Martins

ORCID: https://orcid.org/0000-0002-9107-2485

Universidade Federal do Piauí, Brasil

E-mail:carminhamartins@ufpi.edu.br

\begin{abstract}
Resumo
O desejo por alimentos ocorre para aqueles alimentos que são mais palatáveis, altamente processados, com altos níveis de carboidratos e açúcares refinados e/ou gordura. Já é consolidada a relação direta entre alterações no estado nutricional ideal (como a obesidade) e as doenças cardiovasculares, neurodegenerativas, metabólicas e inflamatórias e o aumento do estresse oxidativo, entretanto, para a análise da qualidade do sono ainda há poucas evidências. Delinear o perfil do estado nutricional, desejo alimentar, marcadores bioquímicos de inflamação e de qualidade sono em profissionais de saúde de um hospital universitário. Este estudo piloto teve caráter transversal, quantitativo e prospectivo. Os participantes recrutados estavam vinculados a um Hospital Universitário Brasileiro. A população alvo foi composta por profissionais de saúde do corpo clínico hospitalar, com admissão realização pelo menos de seis meses anteriores ao início da pesquisa. Foram aplicados questionários para mensuração dos Desejos Intensos por Comida - questionários de Traço e Estado; inflamação, registrados conjuntamente com os dados sociodemográficos e
\end{abstract}


de composição corporal a partir de bioimpedância. Para avaliação do sono, utilizou-se o Índice de Qualidade do Sono de Pittsburgh e da Escala de Sonolência de Epworth. Foram encontradas correlações estatisticamente significativas entre inflamação, qualidade de sono, ângulo de fase e percentual de gordura corporal. Infere-se que os desejos alimentares em grau moderado influenciam na qualidade de sono ruim, perfil bioquímico de inflamação e na composição corporal dos profissionais de saúde. Sugerimos mais estudos de intervenções longitudinais que possam avaliar estas relações à longo prazo.

Palavras-chave: Inflamação; Transtornos do Sono do Ritmo Circadiano; Modalidades alimentares; Impedância elétrica.

\begin{abstract}
Food craving occurs for those foods that are more palatable, highly processed, with high levels of carbohydrates and refined sugars and/or fat. The direct relationship between changes in the ideal nutritional status (such as obesity) and cardiovascular, neurodegenerative, metabolic and inflammatory diseases and the increase in oxidative stress is already consolidated, however, for the analysis of sleep quality, there is still little evidence. To outline the profile of nutritional status, food craving, biochemical markers of inflammation and sleep quality in health professionals from a university hospital. This pilot study was cross-sectional, quantitative and prospective. The recruited participants were linked to a Brazilian University Hospital. The target population consisted of health professionals from the hospital's clinical staff, who had been admitted for at least six months prior to the beginning of the research. Questionnaires were applied to measure Intense Food Cravings - Trait and State questionnaires; inflammation, recorded together with sociodemographic and body composition data from bioimpedance. For sleep assessment, the Pittsburgh Sleep Quality Index and the Epworth Sleepiness Scale were used. Statistically significant correlations were found between inflammation, sleep quality, phase angle and body fat percentage. It is inferred that moderate food cravings influence the quality of poor sleep, biochemical profile of inflammation and body composition of health professionals. We suggest further studies of longitudinal interventions that can assess these long-term relationships.
\end{abstract}

Keywords: Inflammation; Sleep Disorders, Circadian Rhythm; Modalities, alimentary; Electric impedance.

\title{
Resumen
}

El antojo por la comida ocurre por aquellos alimentos que son más sabrosos, altamente procesados, con altos niveles de carbohidratos y azúcares refinados y / o grasas. La relación directa entre alteraciones en el estado nutricional ideal (como la obesidad) y enfermedades cardiovasculares, neurodegenerativas, metabólicas e inflamatorias y el aumento del estrés oxidativo ya está consolidada, sin embargo, para el análisis de la calidad del sueño, aún existe poca evidencia. perfilar el perfil de estado nutricional, ansia por alimentos, marcadores bioquímicos de inflamación y calidad del sueño en profesionales de la salud de un hospital universitario. Este estudio piloto fue transversal, cuantitativo y prospectivo. Los participantes reclutados estaban vinculados a un hospital universitario brasileño. La población diana estuvo constituida por profesionales de la salud del personal clínico del hospital, que habían estado ingresados al menos seis meses antes del inicio de la investigación. Se aplicaron cuestionarios para medir los antojos de alimentos intensos: cuestionarios de rasgo y estado; inflamación, registrada junto con datos sociodemográficos y de composición corporal de bioimpedancia. Para la evaluación del sueño, se utilizaron el índice de calidad del sueño de Pittsburgh y la escala de somnolencia de Epworth. Se encontraron correlaciones estadísticamente significativas entre la inflamación, la calidad del sueño, el ángulo de fase y el porcentaje de grasa corporal. Se infiere que los antojos de alimentos moderados influyen en la calidad del mal sueño, el perfil bioquímico de la inflamación y la composición corporal de los profesionales de la salud. Sugerimos más estudios de intervenciones longitudinales que puedan evaluar estas relaciones a largo plazo.

Palabras clave: Inflamación; Trastornos del Sueño del Ritmo Circadiano; Modalidades alimentarias; Impedancia eléctrica.

\section{Introdução}

Os desejos alimentares são experimentados regularmente por indivíduos com e sem distúrbios alimentares. Normalmente o desejo por alimentos ocorre para aqueles alimentos que são mais palatáveis, altamente processados, com altos níveis de carboidratos e açúcares refinados e/ou gordura (Davidson et al., 2014; De Medeiros et al., 2016; Killgore et al., 2003; Lowe et al., 2018; Whitelock et al., 2018).

O desejo por comida pode ser classificado, conforme as condições que ocorrem, quanto em estado e traço. O traço é considerado uma característica estável dos desejos, mesmo havendo variações das condições temporais e de situações entre indivíduos. Já o estado é sensível às mudanças de estado emocional contextual, psicológico e fisiológico. Em comum possuem, entre outras, fusão cognição e desejos alimentares e decisão (Cepeda-benito et al., 2000).

Mais da metade dos desejos por comida resultam no consumo de alimentos, mesmo nos indivíduos sem distúrbios 
alimentares, podendo ocorrer de 9 a 13 vezes por semana, influenciando diretamente na manutenção do peso corporal bem como as relações do estado nutricional (Knauper et al., 2011).

O estado nutricional pode ser definido como equilíbrio entre a ingestão e as necessidades destes indivíduos em termos de nutrientes. Este equilíbrio é alcançado a partir do suprimento total da necessidade fisiológica dos mais diversos nutrientes, mantendo adequadas as funções corporais. Desnutrição e obesidade são consideradas alterações do estado nutricional, e ambas contribuem para aumento da morbi-mortalidade (Acuña \& Cruz, 2004).

Sabe-se que ácidos graxos livres insaturados estão elevados na circulação sistêmica nos humanos com sobrepeso/ obesidade; e que tecidos com características lipídicas não saturadas podem estar associados à síntese de prostaglandinas, precursoras do processo inflamatório (de Oliveira et al., 2019).

Já é consolidada a relação direta entre alterações no estado nutricional ideal (obesidade) as doenças cardiovasculares, neurodegenerativas, metabólicas e inflamatórias e o aumento do estresse oxidativo, entretanto, para a análise da qualidade do sono ainda há poucas evidências (Daiber et al., 2017; Egea et al., 2017; Ischiropoulos \& Beckman, 2003).

Sabe-se que a ativação da inflamação celular devido à interrupção do sono é existente, apesar de permitir várias vias para tamponamento. Sono perturbado ou frequentemente insuficiente gera estados de ativação imunológica intensificada e, por conseguinte, alterando a sinalização das vias regulatórias do hipocampo, influenciando o estado dos níveis comportamentais bem como a memória espacial (Hunt et al., 2021; Piber, 2021).

O objetivo deste trabalho é delinear o perfil do estado nutricional, desejo alimentar, marcadores bioquímicos de inflamação e de qualidade sono em profissionais de saúde de um hospital universitário.

\section{Metodologia}

Este estudo piloto teve caráter transversal, quantitativo e prospectivo, conforme orientações de Estrela (2018). Os participantes recrutados estavam vinculados a um Hospital Universitário Brasileiro. A população alvo foi composta por profissionais de saúde do corpo clínico hospitalar, com atuação nos postos de internação, Unidades de Terapia Intensiva e ambulatório, com admissão realização pelo menos de seis meses anteriores ao início da pesquisa.

Para o cálculo da amostra será utilizada a proporção mínima padrão de 50\%. Será adotada uma margem de erro de $5 \%$, com intervalo de confiança de $95 \%$. Este trabalho fez parte de uma tese de doutorado, sendo pródromo à realização da pesquisa em sua totalidade. Foi aprovado no CEP local obedecendo todas as prerrogativas legais estabelecidas por meio da resolução CNS 466/2012.

Os critérios de elegibilidade foram: ser profissional de saúde vinculado ao hospital universitário (níveis superior e técnico), de ambos os sexos, com idade entre 20 e 59 anos. Foram excluídos participantes com incapacidade na comunicação (cunho emissivo ou receptivo), grande ganho ou perda de peso (> $5 \mathrm{~kg}$ ) nos últimos seis meses, uso recreativo de drogas psicoativas, uso de cigarro (> 1 cigarro por dia), uso de nicotina (comprimido/ adesivo), trauma ou ferimento recente significativo na cabeça, história de epilepsia, gravidez, diabetes, câncer, doenças cardíacas, diagnóstico prévio autorreferido de distúrbio alimentar ou qualquer distúrbio psiquiátrico.

Foram medidos: Desejos Intensos por Comida - questionários de Traço e Estado (QDIC-T, QDIC-E) validados para o português brasileiro (Cepeda-benito et al., 2000; Ulian et al., 2017a, 2017b); inflamação (a partir de amostra sanguínea, foi aferido o valor via Proteína $C$ reativa $-\mathrm{PCR}$ - ultrassensível), registrados conjuntamente com os dados sociodemográficos e de composição corporal a partir de bioimpedância - utilizado o Inbody S10®, com 4 canais). Para avaliação do sono, utilizou-se o Índice de Qualidade do Sono de Pittsburgh (Pittsburgh Sleep Quality Index - PSQI) e da Escala de Sonolência de Epworth (Epworth Sleepiness Scale - ESE). Os valores de classificação do IMC $(\mathrm{kg} / \mathrm{m} 2)$ segundo o estado nutricional global foram: baixo peso (>18,5), eutrófico (18,5-24,9), sobrepeso (25-29,9), obesidade grau I (30 a 34,5), obesidade II (35 a 39,9), grau III 
$(\geq 40)($ WHO, 2002).

O Questionário de desejo alimentar tem duas formas de medir a característica ou traço habitual (FCQ-T) e o estado (FCQ-S) dos desejos alimentares. O primeiro foi desenvolvido para acessar aspectos dos desejos intensos por comida ao longo do tempo e em diversas situações, considerando-os como um comportamento traço (usual) do respondente. Maiores escores neste questionário se relacionam a um comer mais exagerado. Já o segundo é um instrumento sensível a mudanças de estados contextuais, psicológicos e fisiológicos em resposta a situações específicas (como eventos estressantes, ou de privação alimentar), considerando o desejo intenso por comida como um comportamento estado (esporádico) do respondente (Cepedabenito et al., 2000).

As classificações para o FCQ-T são feitas em uma escala de 6 pontos, com "Nunca" marcou 1 e "Sempre" marcou 6. O FCQ-T é composto por 39 afirmações, cuja pontuação varia de 39 a 214 pontos. Não há pontos de corte para este questionário, mas os escores maiores escores se relacionam ao comer exagerado (Cepeda-benito et al., 2000; Moreno et al., 2008).

A determinação da concentração de PCR no soro será feita segundo o método colorimétrico- enzimático, utilizando kit ELISA (Labtest ${ }^{\circledR}$ ). O processo de análise foi feito de acordo com as recomendações do fabricante. Valores $>0,5 \mathrm{mg} / \mathrm{L}$ serão considerados como indicativo de processos inflamatórios (Heil; Koberstein, 2004).

O PSQI refere-se à qualidade do sono no último mês e fornece um índice de gravidade e natureza do transtorno para a amostra, apresentando excelentes sensibilidade e especificidade, respectivamente de $89,6 \%$ e $86,5 \%$. O escore global varia de 0 a 21, a saber que quanto maior a pontuação, pior a qualidade do sono. Agrupadas em 7 componentes, as 19 questões autorreferenciadas apresentam pesos que variam de 0 a 3 , e indicam grave dificuldade em pelo menos 2 componentes quando a pontuação global >5, ou moderada em mais de 3 componentes.

Já o ESE fornece um índice de probabilidade de cochilar em determinadas situações cotidianas, a partir da avaliação da sonolência diurna recente. Com pesos variando de 0 a 3 em 8 situações, seu escore total pode variar entre 0 e 24 , sendo que escores $>10$ sugerem a existência de Sonolência Diurna Excessiva.

\section{Resultados e Discussão}

Após o período de recrutamento, 53 pacientes foram convocados para realizar as avaliações de composição corporal, qualidade de sono, perfil bioquímico de inflamação e o preenchimento dos questionários de qualidade de sono e sonolência e de desejo alimentar. Foram excluídos: 09 por não comparecerem em todas as provas, 01 por não preencher completamente o questionário referente ao sono e 01 por não ter o resultado do exame bioquímico de inflamação. Totalizando 42 participantes componentes da amostra, com idade média de 36,5 $\pm 6,5$ anos de idade, conforme a Tabela 1 . 
Tabela 1 - Perfil sociodemográfico da amostra, Teresina (PI).

\begin{tabular}{|c|c|}
\hline Variável & $\mathbf{N}(\%)$ \\
\hline \multicolumn{2}{|l|}{ Gênero } \\
\hline Masculino & $09(21,4)$ \\
\hline Feminino & $33(78,6)$ \\
\hline \multicolumn{2}{|l|}{ Idade } \\
\hline$<25$ anos & $03(07)$ \\
\hline Entre 26 e 39 anos & $26(62)$ \\
\hline$>40$ anos & $13(31)$ \\
\hline \multicolumn{2}{|l|}{ Jornada } \\
\hline Exclusivamente diurna & $22(52,4)$ \\
\hline Exclusivamente noturna & $02(4,8)$ \\
\hline Mista & $18(42,9)$ \\
\hline \multicolumn{2}{|l|}{ Escolaridade } \\
\hline$<12$ anos & $06(14,3)$ \\
\hline$>12$ anos & $36(85,7)$ \\
\hline Total & 42 \\
\hline
\end{tabular}

Fonte: Autores (2021).

Os resultados das pontuações obtidas nos questionários de Desejos Intensos por Comida - questionários de Traço e Estado (QDIC-T, QDIC-E). A pontuação mediana total para o QDIC-T foi de 101 pontos, variando de 48,0 a 179. A pontuação total média para o QDIC-E foi de 40, variando de 15 a 71. Isso indica que os participantes da amostra tendem a ceder aos desejos alimentares em grau moderado.

Tabela 2 - Estado nutricional e composição corporal dos participantes, Teresina (PI).

\begin{tabular}{|c|c|}
\hline Variável & $\mathbf{N}(\%)$ \\
\hline \multicolumn{2}{|l|}{ IMC } \\
\hline Eutrófico & $18(42,9)$ \\
\hline Sobrepeso & $20(47,5)$ \\
\hline Obesidade grau I & $02(4,8)$ \\
\hline Obesidade grau II & $02(4,8)$ \\
\hline \multicolumn{2}{|l|}{ Massa Muscular Esquelética } \\
\hline$\leq 20$ & $06(14,3)$ \\
\hline Entre 21 e 29 & $28(66,7)$ \\
\hline$\geq 30$ & $08(19)$ \\
\hline \multicolumn{2}{|l|}{ Ângulo de Fase } \\
\hline Entre 5,4 e 5,99 & $13(31)$ \\
\hline Entre 6 e 10 & $27(64,3)$ \\
\hline Entre 10 e 15 & $02(4,8)$ \\
\hline \multicolumn{2}{|l|}{ Percentual de Gordura Corporal } \\
\hline$<24,9$ & $12(38,6)$ \\
\hline Entre 25 e 29,9 & $06(14,3)$ \\
\hline$>30$ & $24(57,1)$ \\
\hline Total & 42 \\
\hline
\end{tabular}

Fonte: Autores (2021).

A obesidade é caracterizada por uma alteração na morfologia dos adipócitos e infiltração de tecido por monócitos. Após a interação macrófagos/adipócitos há a produção de adipocitocinas, que estão diretamente envolvidas no controle da nutrição, na regulação da sensibilidade à insulina e no metabolismo lipídeo-carboidrato bem como modulação de processos inflamatórios, hemostasia e angiogênese. A leptina é um tipo de adipocina produzida pelos adipócitos em proporção à quantidade de gordura corporal, sendo secretada de forma alterada em indivíduos obesos (Dziegielewska-Gesiak, 2021).

Não obstante, se observa que tanto patologias relacionadas à inflamação de baixo grau como a maioria das doenças 
metabólicas há valores maiores ligados ao aumento de biomarcadores de estresse oxidativo (Daiber et al., 2017; Egea et al., 2017; Ischiropoulos \& Beckman, 2003). A nível celular, outro parâmetro que merece destaque é o ângulo de fase. Classicamente derivado diretamente da relação entre a reactância (capacitância resistiva existente nas membranas celulares) e a resistência (oposição pura do condutor biológico a partir da passagem da corrente elétrica) que os todos os tecidos corporais irão oferecer à passagem de corrente elétrica.

Quanto mais íntegras estiverem as superfícies das membranas, maior será o armazenamento de energia e, por conseguinte, maior será o ângulo de fase formado, por isso, infere-se que ele indica de integridade da membrana celular, fator prognóstico em determinadas patologias, estado geral de saúde do paciente. A variabilidade pode ocorrer dependendo da idade e do sexo, mas de modo geral, indivíduos saudáveis apresentam valores médios de AF entre 4 e 15 graus (García Almeida et al., 2021).

Ao conferir o resultado do PCR ultrassensível dos participantes de nossa pesquisa, foi observado a presença de inflamação de baixo grau na amostra em $57,76 \%(n=23)$ contra 45,23\% (n=19) de dosagens dentro da normalidade. É relevante comentar que grande parte dos participantes estão com aumento do percentual de gordura, associado a altos valores no IMC, nos altos escores do desejo alimentar e baixos relacionados ao sono, como demonstrados na Tabela 3, abaixo.

Tabela 3 - Qualidade de sono e sonolência excessiva diurna, Teresina (PI).

\begin{tabular}{ll|l}
\hline \multicolumn{1}{c|}{ Variável } & \multicolumn{1}{c}{$\mathbf{N}(\%)$} \\
\hline PSQI & & $08(19)$ \\
& Boa & $26(62)$ \\
& Ruim & $08(19)$ \\
& Distúrbio do Sono & \\
\multirow{2}{*}{ ESE } & $<10$ & $26(61,9)$ \\
& $>10$ & $16(38,1)$ \\
\hline Total & $\mathbf{4 2}$ \\
\hline
\end{tabular}

Fonte: Autores (2021).

O sono é caracterizado pela baixa capacidade de resposta a estímulos externos e atividade motora reduzida. Ele pode ser dividido em duas fases principais, que se alternam de forma cíclica: sono sem movimento rápido dos olhos (NREM) e sono REM (sono com o movimento rápido dos olhos). O sono NREM, em humanos, pode ainda ser subclassificado em 3 estágios, estando exatamente relacionado ao aumento da profundidade do sono (estágios N1, N2 e N3). Já o sono REM é mais intenso durante a segunda metade do sono noturno (Hunt et al., 2021; Modeling et al., 2019).

Os achados foram ratificados por meio do coeficiente de correlação de Spearman positiva entre os parâmetros de inflamação versus IMC ( $<<0,02)$; versus massa de gordura $(\mathrm{p}<0,001)$; e negativa quando correlacionada ao ângulo de fase $(\mathrm{p}<0,003)$, ou seja, quanto maior o valor de inflamação obtido menor é a integridade da membrana celular, já a correlação entre PSQI e o ESE não obteve achado estatístico relevante.

As outras variáveis foram analisadas sob a matriz de correlação com a variável independente considerada (desejo), na qual foram obtidos os seguintes achados com correlação positiva: percentual de massa de gordura (0,32), PSQI $(0,39)$, parâmetro bioquímico de inflamação $(0,38)$ e $\operatorname{IMC}(0,3)$. Ainda que fraca houve correlação negativa entre o desejo alimentar e massa muscular esquelética $(-0,23)$ e ângulo de fase $(-0,19)$.

Apesar das diferenças culturais, geográficas e econômicas entre nordeste e sul do Brasil, o perfil de consumo alimentar em profissionais de saúde deste estudo foi muito similar ao encontrado em estudo realizado em Pelotas/RS. Apesar de não ter utilizado um questionário sobre a qualidade do sono, encontrou que do total da amostra, 75,3\% relataram dormir menos de 8 horas diárias, quantidade insuficiente de sono recomendada para adultos. Também foi encontrado também um alto 
consumo de alimentos fortemente associados com o desejo alimentar, pois os entrevistados refeririam consumir regularmente doces, refrigerantes e sucos industrializados e frituras e gordura aparente das carnes, respectivamente, $43,4 \%$ e $47 \%$. Cabe ressaltar que estes alimentos que possuem alta palatabilidade também são constantemente alvo de desejos alimentares intensos, além de possuir um alto índice calórico e de gorduras trans, contribuindo para o surgimento de sobrepeso e obesidade (Schumann Kruger et al., 2018).

Demonstrou-se que o desejo alimentar estado ou traço estão positivamente associado à alimentação emocional (comer excessivamente em resposta às emoções distintas do desejo alimentar), incluindo fusão cognitiva a desejos alimentares, aceitação da presença de desejos alimentares e vontade de não agir sobre eles, e preocupação alimentar, influenciando diretamente na manutenção do peso corporal bem como as relações do estado nutricional (Cepeda-benito et al., 2000; Moreno et al., 2008; Ulian et al., 2017a, 2017b).

Considerando que boa parte da amostra trabalha de forma noturna, restrição crônica de sono, dentre outros tantos efeitos fisiológicos, também pode implicar no aumento dos desejos por alimentos pouco saudáveis; e, podem ser experimentados de forma diferente devido a alterações na função gustativa quando experimentados os sabores predominamente umami ou azedo. Ou seja, a falta de sono pode influenciar nas decisões e comportamento alimentar, agindo ainda que parcialmente por meio do nosso paladar (Lv et al., 2018).

\section{Conclusão}

Infere-se que os desejos alimentares em grau moderado influenciam na qualidade de sono ruim, perfil bioquímico de inflamação e na composição corporal dos profissionais de saúde. Sugerimos mais estudos de intervenções longitudinais que possam avaliar estas relações à longo prazo.

\section{Referências}

Acuña, K., \& Cruz, T. (2004). Avaliação do estado nutricional de adultos e idosos e situação nutricional da população brasileira. Arquivos Brasileiros de Endocrinologia \& Metabologia, 48(3), 345-361. https://doi.org/10.1590/s0004-27302004000300004

Cepeda-benito, A., Gleaves, D. H., Williams, T. L., \& Erath, S. A. (2000). The Development and Validation of the State and Trait Food-Cravings Questionnaires. Behavior Therapy, a 31, 151-173. https://doi.org/10.1016/S0005-7967(99)00141-2

Daiber, A., Steven, S., Weber, A., Shuvaev, V. V, \& Vladimir, R. (2017). Review for Themed Issue 'Redox biology and oxidative stress in health and disease' Targeting vascular ( endothelial ) dysfunction. Br J Pharmacol. https://doi.org/10.1111/bph.13517

Davidson, T. L., Tracy, A. L., Schier, L. A., \& Swithers, S. E. (2014). A view of obesity as a learning and memory disorder. Journal of Experimental Psychology: Animal Behavior Processes, 40(3), 261-279. https://doi.org/10.1037/xan0000029

De Medeiros, Q., Yamamoto, M. E., Cec1, A., Fatima, L., Pedrosa, C., \& Simon, C. (2016). The Brazilian version of the three-factor eating questionnaireR21 : psychometric evaluation and scoring pattern. https://doi.org/10.1007/s40519-016-0256-X

de Oliveira, C., de Freitas, J. S., Macedo, I. C., Scarabelot, V. L., Ströher, R., Santos, D. S., Souza, A., Fregni, F., Caumo, W., \& Torres, I. L. S. (2019). Transcranial direct current stimulation (tDCS) modulates biometric and inflammatory parameters and anxiety-like behavior in obese rats. Neuropeptides, 73(July 2018), 1-10. https://doi.org/10.1016/j.npep.2018.09.006

Dziegielewska-Gesiak, S. (2021). Metabolic syndrome in an aging society - role of oxidant-antioxidant imbalance and inflammation markers in disentangling atherosclerosis. Clinical Interventions in Aging, 16(April), 1057-1070. https://doi.org/10.2147/CIA.S306982

Egea, J., Fabregat, I., Frapart, Y. M., Ghezzi, P., Görlach, A., Kietzmann, T., Kubaichuk, K., Knaus, U. G., Lopez, M. G., Olaso-gonzalez, G., Petry, A., Schulz, R., Vina, J., Winyard, P., Abbas, K., Ademowo, O. S., Afonso, C. B., Andreadou, I., Antelmann, H., ... Daiber, A. (2017). Redox Biology European contribution to the study of ROS : A summary of the fi ndings and prospects for the future from the COST action BM1203 ( EU-ROS ). Redox Biology 13, 13(May), 94-162. https://doi.org/10.1016/j.redox.2017.05.007

Estrela, C. (2018). Metodologia Científica: Ciência, Ensino, Pesquisa. Editora Artes Médicas.

García Almeida, J. M., García García, C., Vegas Aguilar, I. M., Bellido Castañeda, V., \& Bellido Guerrero, D. (2021). Morphofunctional as sessment of patient nutritional status: a global approach. Nutrición Hospitalaria. https://doi.org/10.20960/nh.03378

Hunt, C. A., Smith, M. T., Mun, C. J., Irwin, M. R., \& Finan, P. H. (2021). Trait positive affect buffers the association between experimental sleep disruption and inflammation. Psychoneuroendocrinology, 129(December 2020), 105240. https://doi.org/10.1016/j.psyneuen.2021.105240 
Research, Society and Development, v. 10, n. 14, e245101422419, 2021

(CC BY 4.0) | ISSN 2525-3409 | DOI: http://dx.doi.org/10.33448/rsd-v10i14.22419

Ischiropoulos, H., \& Beckman, J. S. (2003). Oxidative stress and nitration in neurodegeneration : Cause, effect, or association? J. Clin. Invest., 111(2), 163169. https://doi.org/10.1172/JCI200317638.Oxidation

Killgore, W. D. S., Young, A. D., Femia, L. A., Bogorodzki, P., Rogowska, J., \& Yurgelun-Todd, D. A. (2003). Cortical and limbic activation during viewing of high- versus low-calorie foods. Neurolmage, 19(4), 1381-1394. https://doi.org/10.1016/S1053-8119(03)00191-5

Knauper, B., Pillay, R., Lacaille, J., Mccollam, A., \& Kelso, E. (2011). Replacing craving imagery with alternative pleasant imagery reduces craving. Appetite, 57, 173-178. https://doi.org/10.1016/j.appet.2011.04.021

Lowe, C. J., Staines, W. R., Mannochio, F., \& Hall, P. A. (2018). The neurocognitive mechanisms underlying food cravings and snack food consumption. A combined continuous theta burst stimulation (cTBS) and EEG study. NeuroImage, 177, 45-58. https://doi.org/10.1016/j.neuroimage.2018.05.013

Lv, W., Finlayson, G., \& Dando, R. (2018). Sleep, food cravings and taste. Appetite, 125, 210-216. https://doi.org/10.1016/j.appet.2018.02.013

Modeling, L. M., Measurement, F., Snowrift, O. N., Environmental, A. R., Regional, S., Power, E., Limited, G. C., Influence, T. H. E., Snow, O. F., On, F., Around, S., Embankment, T. H. E., Wind, I. N., Snow, B., Aspect, O. F., On, R. R., Distribution, S., Model, A. A. C., Simulation, N., \& Maciejowski, J. M. (2019). No 主観的健康感を中心とした在宅高齢者における 健康関連指標に関する共分散構造分析Title. Journal of Wind Engineering and Industrial Aerodynamics, 26(3), 1-4. https://doi.org/10.1007/s11273-020-09706-3\%0Ahttp://dx.doi.org/10.1016/j.jweia.2017.09.008\%0Ahttp s://doi.org/10.1 016/j.energy.202 0.117919\%0Ahttps://doi.org/10.1016/j.coldregions.2020.103116\%0Ahttp://dx.doi.org/10.1016/j.jweia.2010.12.004\%0Ahttp://dx.doi.o

Moreno, S., Fernandez, M. C., \& Cepeda-benito, A. (2008). Clinical Validation of the Trait and State Versions of the Food Craving Questionnaire. 15(3), 375387. https://doi.org/10.1177/1073191107312651

Piber, D. (2021). The role of sleep disturbance and inflammation for spatial memory. Brain, Behavior, \& Immunity - Health, 17(March), 100333. https://doi.org/10.1016/j.bbih.2021.100333

Schumann Kruger, M., Doumid Borges Pretto, A., Bezerra Massaut, K., \& Nunes Moreira, Â. (2018). Evaluation of life habits and eating health professionals in a hospital in the city of south of Brazil. Nutricion Clinica y Dietetica Hospitalaria, 38(4), 204-208. https://doi.org/10.12873/384kruger

Ulian, M. D., Sato, P. D. M., Benatti, F. B., De Campos-Ferraz, P. L., Roble, O. J., Unsain, R. F., Gualano, B., \& Scagliusi, F. B. (2017a). Cross-cultural adaptation of the state and trait food cravings questionnaires (FCQ-S and FCQ-T) into Portuguese. Ciencia e Saude Coletiva, 22(2), 403-416. https://doi.org/10.1590/1413-81232017222.18272015

Whitelock, V., Nouwen, A., van den Akker, O., \& Higgs, S. (2018). The role of working memory sub-components in food choice and dieting success. Appetite, 124, 24-32. https://doi.org/10.1016/j.appet.2017.05.043

WHO. (2002). The World Health Organization Report 2002: reducing risks, promoting healthy life. WHO Library Cataloguing-in Publication Data, 232. 